\title{
Nuclear Star Clusters across the Hubble Sequence
}

\author{
Torsten Böker \\ European Space Agency, Dept. RSSD, Keplerlaan 1, 2200 AG Noordwijk, \\ Netherlands tboeker@rssd.esa.int
}

Summary. Over the last decade, HST imaging studies have revealed that the centers of most galaxies are occupied by compact, barely resolved sources. Based on their structural properties, position in the fundamental plane, and spectra, these sources clearly have a stellar origin. They are therefore called "nuclear star clusters" (NCs) or "stellar nuclei". NCs are found in galaxies of all Hubble types, suggesting that their formation is intricately linked to galaxy evolution. In this contribution, I briefly review the results from recent studies of NCs, touch on some ideas for their formation, and mention some open issues related to the possible connection between NCs and supermassive black holes.

\section{Introduction}

The nuclei of galaxies are bound to provide "special" physical conditions because they are located at the bottom of the potential well of their host galaxies. This unique location manifests itself in various distinctive phenomena such as active galactic nuclei (AGN), central starbursts, or extreme stellar densities. The evolution of galactic nuclei is closely linked to that of their host galaxies, as inferred from a number of global-to-nucleus scaling relations discovered in the last decade.

Recently, observational and theoretical interest has been refocused onto the compact and massive star clusters found in the nuclei of galaxies of all Hubble types. Historically, the nuclei of $\mathrm{dE}, \mathrm{N}$ galaxies have been best studied, but it has become clear from recent HST studies that similar objects exist also in normal spirals and ellipticals. At face value, these "nuclear star cluster: ${ }^{1}$ ' are an intriguing environment for the formation of massive black holes because of their extreme stellar density. NCs may also constitute the

\footnotetext{
${ }^{1}$ A word on terminology: historically, the compact light excess found in the centers of "nucleated" early-type galaxies is often referred to as a "stellar nucleus". In most studies of disk-dominated galaxies, however, the term "nuclear star cluster" is used. Because we now know that in terms of size and luminosity, both types of objects are indistinguishable, and likely differ only their in evolutionary stage, it seems reasonable to adopt a single term for them. Since "star cluster" seems more descriptive of the nature of these objects than "nucleus" (after all, every galaxy has a nucleus, i.e. a center), we will refer to them as "nuclear star clusters" or NCs for all galaxies types.
} 


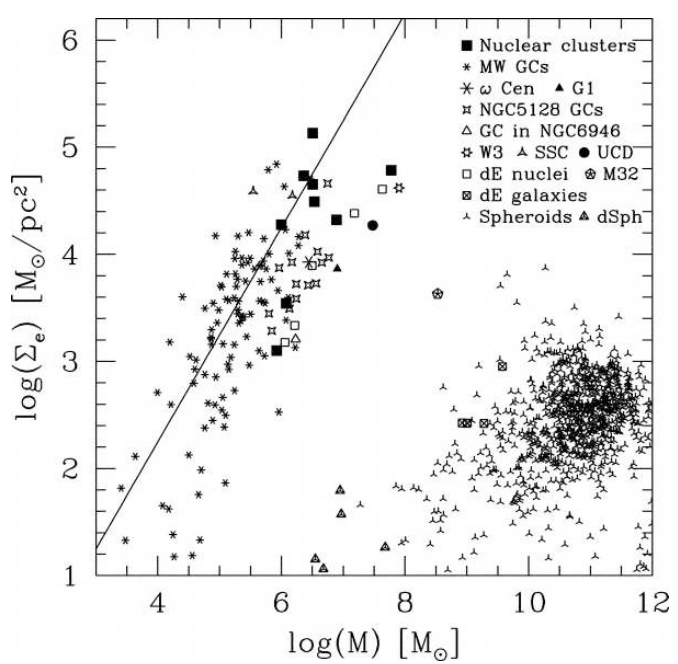

Fig. 1. Mean projected mass density of various stellar systems inside their effective radius $r_{e}$, plotted against their total mass. This is similar to a face-on view of the fundamental plane. NCs occupy the high end a region populated by other types of massive stellar clusters, and are well separated from elliptical galaxies and spiral bulges. The solid line represents a constant cluster size, i.e. $r_{e}=3$ pc (from [19]).

progenitors of at least some halo globular clusters via "NC capture" following the tidal disruption of a satellite galaxy. Finally, their formation process is influenced by (and important for) the central potential, which in turn governs the secular evolution of their host galaxies. In what follows, I briefly summarize what has been learned about NCs over the last few years.

\section{Properties of Nuclear Star Clusters}

Extragalactic star clusters are compact sources, and in general, their study requires space-based resolution. It doesn't come as a surprise, therefore, that the HST has been instrumental for recent progress in the understanding of NCs. Over the last decade, a number of HST studies - both via imaging and spectroscopic observations - have contributed to the following picture of NCs:

1) NCs are common: the fraction of galaxies with an unambiguous NC detection is $75 \%$ in late-type (Scd-Sm) spirals [1, 50\% in earlier-type (Sa-Sc) spirals [4], and $70 \%$ in spheroidal (E \& S0) galaxies [5]. All these numbers are likely lower limits, although for different reasons. In the latest-type disks, it is sometimes not trivial to locate the galaxy center unambiguously so that no particular source can be identified with it. In contrast, many early-type galax- 
ies have very steep surface brightness profiles (SBPs) that make it difficult to detect even luminous clusters against this bright background.

2) NCs are much more luminous than "normal" globular clusters (GCs). With typical absolute I-band magnitudes between -14 and -10 [1, 5], they are roughly 40 times more luminous than the average MW globular cluster [10].

3) However, NCs are as compact as MW GCs. Their half-light radius typically is $2-5 \mathrm{pc}$, independent of galaxy type $2,2,5$,

4) Despite their compactness, NCs are very massive: their typical dynamical mass is $10^{6}-10^{7} \mathrm{M}_{\odot}[19$ which is at the extreme high end of the GC mass function.

5) Their mass density clearly separates NCs from compact galaxy bulges. This is demonstrated in Figure 1 which compares the mass and mass density of NCs to that of other spheroidal stellar systems. The clear gap between bulges/ellipticals on the one hand, and NCs on the other hand makes a direct evolutionary connection between the two classes of objects unlikely.

6) The star formation history of NCs is complex, as evidenced by the fact that most NCs have stellar populations comprised of multiple generations of stars 20, 13. The youngest generation is nearly always younger than $100 \mathrm{Myr}$ which is strong evidence that NCs experience frequent and repetitive star formation episodes [20.

7) Due to three recent and independent studies of NCs in different galaxy types [13, 21, 5, it has become clear that NCs obey similar scaling relationships with host galaxy properties as do supermassive black holes (SMBHs). As an example, Figure 2 shows the NC mass as a function of bulge luminosity. While the implications of this result are not yet clear (see $\S 4$ ), these studies have renewed interest in NCs because of the potentially important role that NCs play in the evolution of their host galaxies.

\section{How (and when) do Nuclear Clusters Form?}

The processes that funnel gas onto NCs in the local universe have recently been studied in some detail, enabled by significant improvements to the sensitivity and spatial resolution of mm-interferometers. Figure 3 shows the molecular gas distribution in the nearby spiral NGC 6946. Both the morphology and the kinematics of the gas can be well explained by the effects of a smallscale stellar bar. The S-shaped flow pattern onto the nucleus and the large $\left(1.6 \cdot 10^{7} \mathrm{M}_{\odot}\right)$ gas concentration in the inner $\approx 10 \mathrm{pc}$ lend credibility to the "repetitive burst" scenario for NC growth.

Less clear, however, are the reasons for why gas accumulates in the nucleus of a shallow disk galaxy in the absence of a prominent central mass concentration, i.e. how the "seed clusters" form initially. A few studies have attempted to provide an explanation for this puzzle. For example, [12] suggests the 


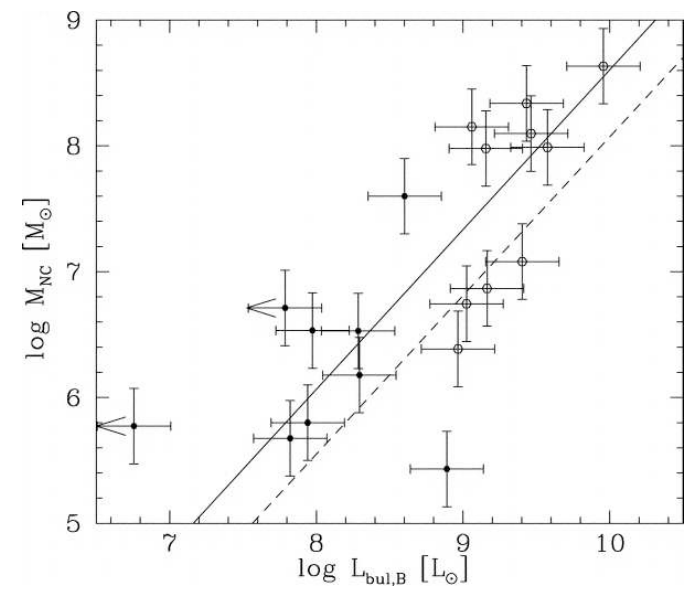

Fig. 2. Relation between $\mathrm{NC}$ mass in spiral galaxies and bulge luminosity $\log \left(\mathrm{L}_{\mathrm{B}}\right)\left[\mathrm{L}_{\odot}\right]$ of the host galaxy (from [13]). Open symbols denote early-type spirals, and filled symbols denote late-type spirals. There is a strong correlation in the sense that galaxies with more luminous bulges have more massive NCs. The solid line indicates the best linear fit to the data, while the dashed line indicates the relation between the SMBH mass and bulge luminosity for the sample of [11].

magneto-rotational instability in a differentially rotating gas disk as a viable means to transport gas towards the nucleus and to support (semi)continuous star formation there.

More recently, 6] have pointed out that the tidal field becomes compressive in shallow density profiles, causing gas to collapse onto the nucleus of a disk galaxy. If correct, then $\mathrm{NC}$ formation is indeed expected to be a natural consequence of galaxy formation, which would go a long way towards explaining at least some of the observed scaling relations between NCs and their host galaxies.

The question of when a particular NC (i.e. its "seed" cluster) has formed is equivalent to asking how old its oldest stars are. This question is extremely difficult to answer in all galaxy types, albeit for different reasons. In late-type spirals, for example, the NC nearly always contains a young stellar population which dominates the spectrum and thus makes the detection of an underlying older population challenging, not to mention its accurate age determination.

Early-type, spheroidal galaxies, on the other hand, lack the large gas reservoirs of spirals, and thus should experience less frequent nuclear starbursts. One therefore would expect their NCs to contain fewer and older stellar populations. However, early-type galaxies have much steeper surface brightness profiles, and therefore a low contrast between NC and galaxy body. This makes spectroscopic studies of NCs in E's and S0's exceedingly difficult. The few published studies have focused on the $\mathrm{NCs}$ of $\mathrm{dE}, \mathrm{N}$ galaxies, and have 

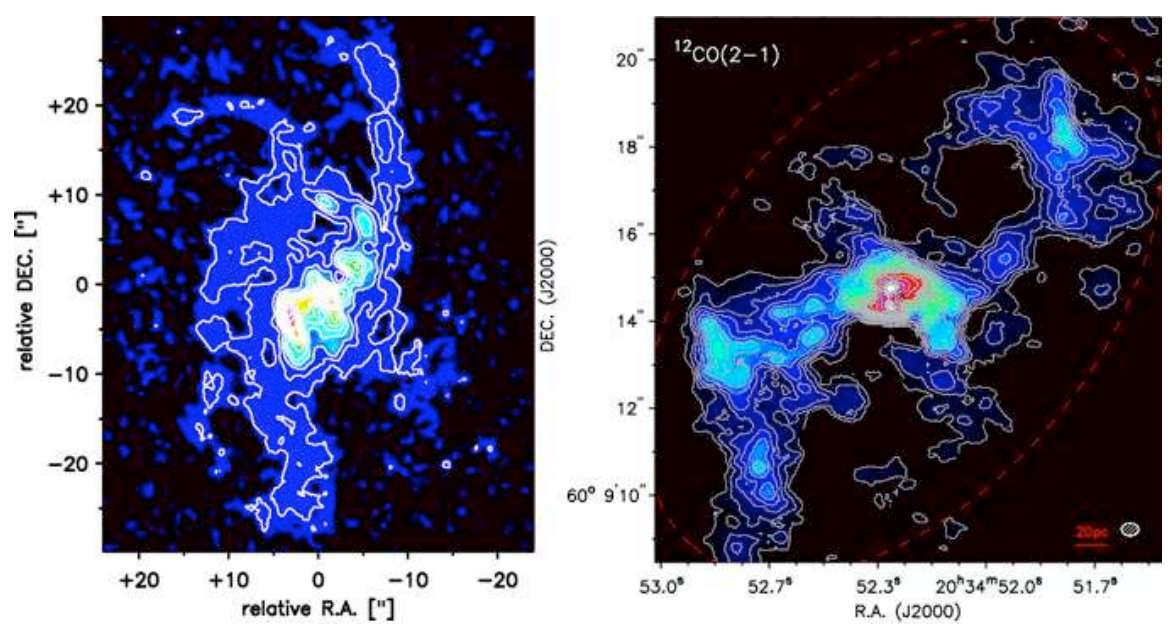

Fig. 3. Left: intensity map of the ${ }^{12} \mathrm{CO}(1-0)$ line in the central $1^{\prime}(1.6 \mathrm{kpc})$ in the nearby spiral galaxy NGC 6946 [16]. Right: higher resolution $\left(0.4^{\prime \prime}\right)$ map of the ${ }^{12} \mathrm{CO}(2-1)$ emission in the central $300 \mathrm{pc}$. Note the S-shaped spiral that appears to "funnel" molecular gas towards the central $10 \mathrm{pc}$ where about $1.6 \cdot 10^{7} \mathrm{M}_{\odot}$ of molecular gas have accumulated [17.

shown that even these can have rather young (few hundred Myrs) stars, as demonstrated e.g. by [3] in the case of NGC 205. Generally speaking, however, most NCs in dE,N galaxies have integrated colors that - while different from those of their host galaxies - are generally consistent with evolved stellar populations at least 1 Gyr old [18]. Considering that there may be even older stellar populations "hiding", this age should only be considered a lower limit for the oldest stellar population in $\mathrm{dE}, \mathrm{N}$ nuclei.

In fact, it is not implausible that the "seed clusters" for present-day NCs were in place very early in the universe. The average star formation rate over the last $100 \mathrm{Myr}$ in NCs of late-type spirals is $2 \cdot 10^{-3} \mathrm{M}_{\odot} / \mathrm{yr}$ [20]. Assuming this SFR was constant for the past $10 \mathrm{Gyr}$, one would expect a stellar mass of $\approx 2 \cdot 10^{7} \mathrm{M}_{\odot}$ which is within a factor of 4 from the typical NC mass of $5 \cdot 10^{6} \mathrm{M}_{\odot}$ [19]. Turning the argument around, if NCs indeed build up their entire present-day mass via a series of repetive starbursts, then they must have been in place at least $3 \mathrm{Gyr}$ ago, unless their SFRs were significantly higher in the past than over the last $100 \mathrm{Myr}$. Given that observations todate are somewhat biased towards more luminous NCs which likely have a timeaveraged SFR higher than the "typical" NC, this estimate might even be too low. 


\section{A new Paradigm?}

It has recently been proposed by [7] that NCs extend the well-known scaling relation between the mass of a galaxy and that of its central super-massive black hole $(\mathrm{SMBH})$ to lower masses. This has triggered speculation about a common formation mechanism of NCs and SMBHs, being governed mostly by the mass of the host galaxy. The idea put forward is that NCs and SMBHs are two incarnations of a "central massive object" (CMO) which forms in every galaxy. In galaxies above a certain mass threshold $\left(\approx 10^{10} \mathrm{M}_{\odot}\right)$, galaxies form predominantly SMBHs while lower mass galaxies form NCs.

While tantalizing, this apparent connection opens more questions than it answers. For example, we know that some galaxies contain both an NC and a SMBH. A well-known example is the "mini-Seyfert" NGC 4395 [8], but others have been found recently [14, 15]. Why then do some NCs contain SMBHs, but not all? Why do some galaxies apparently contain neither NC nor SMBH? Is an NC possibly a pre-requisite for the formation of a SMBH? Is the formation of a $\mathrm{BH}$ (not necessarily a super-massive one) a logical consequence of the high stellar densities present in NCs? Progress along these lines will require a better understanding of the formation of "pure" disk galaxies in the early universe, as well as improved models for the evolution of extremely dense stellar systems.

\section{References}

1. Böker, T. et al. 2002, AJ, 123, 1389

2. Böker, T. et al. 2004, AJ, 127, 105

3. Butler, D. J. \& Martínez-Delgado, D. 2005, AJ, 129, 2217

4. Carollo, C. M. et al. 1997, AJ, 114, 2366

5. Côté, P., et al. 2006, ApJS, 165, 57

6. Emsellem, E. \& van de Ven, G. 2007, ApJ, submitted

7. Ferrarese, L. et al. 2006, ApJ, 644, L21

8. Filippenko, A. V. \& Ho, L. C. et al. 2003, ApJ, 588, L13

9. Geha, M., Guhathakurta, P., \& van der Marel, R. P. 2002, AJ, 124, 3073

10. Harris, W. E. 1996, AJ, 112, 1487

11. Marconi, A., \& Hunt, L. K. 2003, ApJ, 589, L21

12. Milosavlević, M. 2004, ApJ, 605, L13

13. Rossa, J. et al. 2006, AJ, 132, 1074

14. Satyapal, S. et al. 2007, ApJ, 663, L9

15. Shields, J. C. et al. 2007, ApJ, submitted

16. Schinnerer, E. et al. 2006, ApJ, 649, 181

17. Schinnerer, E. et al. 2007, A\&A, 462, L27

18. Stiavelli, M. et al. 2001, AJ, 121, 1385

19. Walcher, C. J., et al. 2005, ApJ, 618, 237

20. Walcher, C. J. et al. 2006, ApJ, 649, 692

21. Wehner, E. H. \& Harris, W. E. 2006, ApJ, 644, L17 